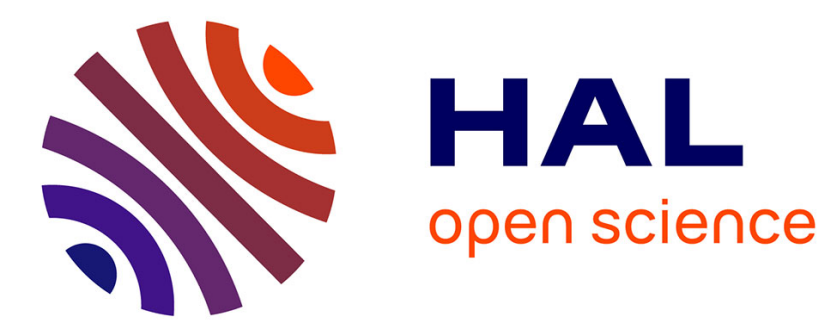

\title{
Triple line dynamics in superfluid helium
}

Françoise Brochard-Wyart

\section{To cite this version:}

Françoise Brochard-Wyart. Triple line dynamics in superfluid helium. Journal de Physique II, 1993, 3 (1), pp.21-26. 10.1051/jp2:1993108 . jpa-00247810

\section{HAL Id: jpa-00247810 https://hal.science/jpa-00247810}

Submitted on 1 Jan 1993

HAL is a multi-disciplinary open access archive for the deposit and dissemination of scientific research documents, whether they are published or not. The documents may come from teaching and research institutions in France or abroad, or from public or private research centers.
L'archive ouverte pluridisciplinaire HAL, est destinée au dépôt et à la diffusion de documents scientifiques de niveau recherche, publiés ou non, émanant des établissements d'enseignement et de recherche français ou étrangers, des laboratoires publics ou privés. 
Classification

Physics Abstracts

$67.70-68.10$

\title{
Short Communication
}

\section{Triple line dynamics in superfluid helium}

\author{
Françoise Brochard-Wyart
}

Institut Curie, 11 rue Pierre et Marie Curie, 75231 Paris Cedex 05, France

(Received 14 September 1992, accepted in final form 28 October 1992)

\begin{abstract}
Superfluid ${ }^{4}$ He fails to wet any substrate, as has been shown theoretically and experimentally. We study some hydrodynamic features expected in partial wetting (in conditions where exchanges with the vapor are negligible): i) the dynamics of dewetting: thin films deposited on non wettable surfaces are metastable below a critical thickness and evolve by nucleation and growth of dry holes. The holes are predicted here to open at constant velocity. ii) The collective modes of a contact line, "triplons", which are propagative.
\end{abstract}

\section{Introduction.}

It has been shown very recently that superfluid ${ }^{4}$ He fails to wet all substrates. Theoretically, Cheng et al. [1] predicted that, at $T=0,{ }^{4} \mathrm{He}$ deposited on weak binding substrates such as the alkali metals could exhibit, depending on the substrate potential, either non wetting or wetting: finite contact angles are predicted for ${ }^{4} \mathrm{II}$ e deposited on potassium, rubidium and cesium, while complete wetting is predicted for lithium, sodium and hydrogen. In a more recent paper, they explore the temperature dependence of the wetting-non wetting phase diagram. Observations by Nacher and Dupont-Roc [2] of heat. flow in ${ }^{4}$ Ile films in a glass tube, with and without an interior cesium ring show that ${ }^{4} \mathrm{He}$ does not wet cesium. However wetting is observed for $\mathrm{Rb}$ and $\mathrm{K}$ rings. Using vapor pressure isotherm measurements, Mukherjee, Druist and Chan [3] indicate a complete absence of ${ }^{4} \mathrm{Ile}$ adsorption on cesium coated graphite for $T \leq 2 \mathrm{~K}$. Ketola, Wang and Hallock [4] (using third sound) have observed anomalous wetting of partly Cs-coated glass. Increasing the vapor pressure, they monitor a transition between dry cesium and cesium covered by a thin film, whereas Nacher observed no wetting for ${ }^{4} \mathrm{He}$ in coexistence with bulk liquid. This discrepency has been attributed to roughness and/or surface impurities (e.g.: oxygen promoting wetting), which are different in the two experiments. In a more recent work, Rutledge and Taborek [5] were able to prepare very smooth cesium evaporated films onto the surface of a quartz microbalance and show that ${ }^{4} \mathrm{He}$ film undergoes a wetting transition and the associated prewetting transition. 
Our aim in the present note is to study the dynamics of wetting by superfluid helium, assuming: a) a finite contact angle $\theta_{\mathrm{E}}$, (or a negative spreading coefficient $S=\gamma_{\mathrm{so}}-\left(\gamma_{\mathrm{sL}}+\gamma\right)$, where $\gamma_{i j}$ are the bare solid, solid/liquid and liquid/vapor interfacial tensions, and b) negligible exchanges between the liquid and the vapor (thus restricting our attention to very low temperatures).

If a helium film is deposited on a non-wettable substrate, the film is unstable below a critical thickness [6]

$$
e_{\mathrm{c}}=2 \kappa^{-1} \sin \frac{\theta_{\mathrm{E}}}{2}
$$

where $\kappa^{-1}$ is the capillary length.

$$
\kappa^{-1}=\sqrt{\frac{\gamma}{\rho g}}
$$

with $\gamma=0.35 \mathrm{mN} \cdot \mathrm{m}^{-1}, \rho=0.14 \mathrm{~g} \cdot \mathrm{cm}^{-3}$, one expects $\kappa^{-1} \sim 0.5 \mathrm{~mm}$. "Thick" films are metastable: they dewet by nucleation and growth of dry patches [6, 7]. Microscopic films are unstable and dewet by a mechanism of spinodal decomposition, where capillary waves are amplified [8-10]. The dewetting of viscous liquids has been studied recently both experimentally and theoretically: dry holes in the film open and a liquid rim is formed at the periphery of the dry region. The velocity of $\operatorname{rim} V_{\mathrm{d}} \frac{\gamma}{\eta}=\left(\theta_{\mathrm{E}}\right)^{3}$ is constant in time, inversely proportional to the viscosity $\eta$ of the liquid and independent of the initial thickness $e^{6}$. In section 2 we study the dewetting velocity and the shape of the moving rim for inertial liquids.

In section 3 , we consider a contact line between a solid, liquid helium and vapor which is deformed sinusoïdally. Many capillary problems involve the deformations of a contact line: for instance the separation of the line from an anchoring site can be analysed once undulation modes are understood. These modes have been studied for viscous liquids by Ondarçuhu and Veyssié [11]. With silicone oils they found single exponential relaxations, suggestive of purely viscous regimes. We have calculated these collective mode relaxations for viscous liquids [12]. Our aim in section 3 is to discuss the case of inertial liquids. Most of our discussion in section 3 is only qualitative, and ignores all numerical prefactors.

\section{Dewetting: nucleation and growth of dry regions.}

When a liquid film is deposited on a non-wettable surface, dry regions nucleate and grow. We have pictured in figure 1 the opening of a dry patch of a radius $R(t)$ surrounded by a liquid rim, which moves at velocity $U=\frac{\mathrm{d} R}{\mathrm{~d} t}$ The native film is immobile. The liquid of the dry region is collected in the rim of volume $\Omega(t)$ increasing with time. We can simply derive the motion equation of the moving rim from the transfer of surface energies into kinetics energy of the rim.

$$
\frac{1}{2} \rho \Omega U^{2}=-S A
$$

where $A=\pi R^{2}$ is the surface of the dry surface of radius $R$ and $\Omega,(\Omega=A \mathrm{e})$, the volume of the liquid which has been collected in the rim. From equation (3), one finds the velocity $U$ :

$$
U=\sqrt{\frac{2 S}{\rho e}}=\sqrt{g e_{\mathrm{c}}} \sqrt{\frac{e_{\mathrm{c}}}{e}} .
$$




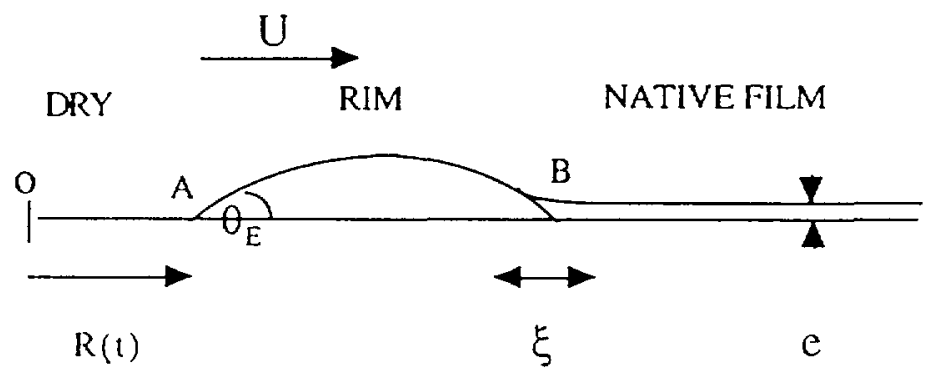

Fig. 1. - Opening of a dry patch of radius $R(t)$ in a helium film of thickness $e$. The liquid is collected in a rim of circular shape. $\theta_{\mathrm{E}}$ is the equilibrium contact angle. $U$ is the dewetting velocity.

The velocity is constant with time! The driving force acting on the rim is constant, but the mass of the rim increases and the resulting motion is uniform. Equation (3) assumes that $e \ll e_{\mathrm{c}}$. In the general case, one must add a gravity energy contribution, which leads to:

$$
U=\sqrt{g \frac{e_{\mathrm{c}}^{2}-e^{2}}{e}} .
$$

Typically, with $S \sim \gamma$, one expects $U \sim \mathrm{cm} \mathrm{s}^{-1}$ for $\frac{e_{\mathrm{c}}}{e} \sim 10$.

The velocity of dewetting is similar to the velocity of opening of a hole in a soap film [13, 14]. The case of soap films is complicated by the presence of the surfactant at the free surface, which gives rise to a relatively complex elastic behavior. In our case, the interfacial tension $\gamma$ is constant, and equation (4) is exact.

The shape of rim is deduced from the motion equation of a liquid sheet. In the lubrication approximation, the velocity $V(z)$ of a liquid sheet of height $\zeta(x)$ is independent of $z$ (plug flow) and the Stokes equation for a non-viscous liquid is:

$$
\rho \zeta\left(\frac{\mathrm{d} V}{\mathrm{~d} t}+V \frac{\mathrm{d} V}{\mathrm{~d} x}\right)=\zeta \gamma \zeta^{\prime \prime \prime}
$$

The derivative of the momentum is balanced by the Laplace pressure gradient. The equation has to be modified at the two contact lines $A$ and $B$ with the solid substrate and the native film, respectively. Because the acceleration term is negligible for $z \rightarrow 0$, the static equilibrium conditions hold at both ends of the rim

$$
\left\{\begin{array}{l}
\theta\left(x_{A}\right)=\theta_{E} \\
\theta\left(x_{B}\right)=0
\end{array}\right\} .
$$

The rim moves at velocity $U$ given by equation (4), except in a narrow region where the velocity slows down to zero. Equation (5) then shows that, in most of the rim, $\zeta^{\prime \prime \prime}=0$, i.e. the pressure is constant and the rim profile is a portion of a perfect circle, except near the contact line $\mathbf{B}$ in a thin region of extension $\xi$. The healing length $\xi$ is deduced from equation (5) by a scaling argument

$$
\rho \frac{U^{2}}{\xi} \cong \gamma \frac{e}{\xi^{3}}
$$

i.e.

$$
\xi \cong \sqrt{\frac{\gamma e}{\rho U^{2}}}=e \sqrt{\frac{\gamma}{2 S}} .
$$


Integrating equation (5) between $A$ and $B$ leads to equation (4). We have pictured in figure 1 the shape of the rim and the small region where $\theta$ falls to zero.

\section{Collective modes of a contact line.}

We consider the contact line a non-wettable solid, superfluid ${ }^{4} \mathrm{He}$ and its vapor (of negligible pressure). The line is deformed sinusoidally with a wavelength $\frac{2 \Pi}{q}$ (Fig. 2). We discuss the resulting oscillation assuming a small contact angle $\theta_{\mathrm{E}} \ll 1$. In this limit, the capillary energy of the weakly disturbed line, with amplitude $u_{q}$ for a wave vector $q$, has been calculated first in reference [15] and is of the form:

$$
f_{\mathrm{e}]} q=\frac{1}{2} \gamma \theta_{\mathrm{E}}^{2}|q|\left|u_{q}\right|^{2}
$$

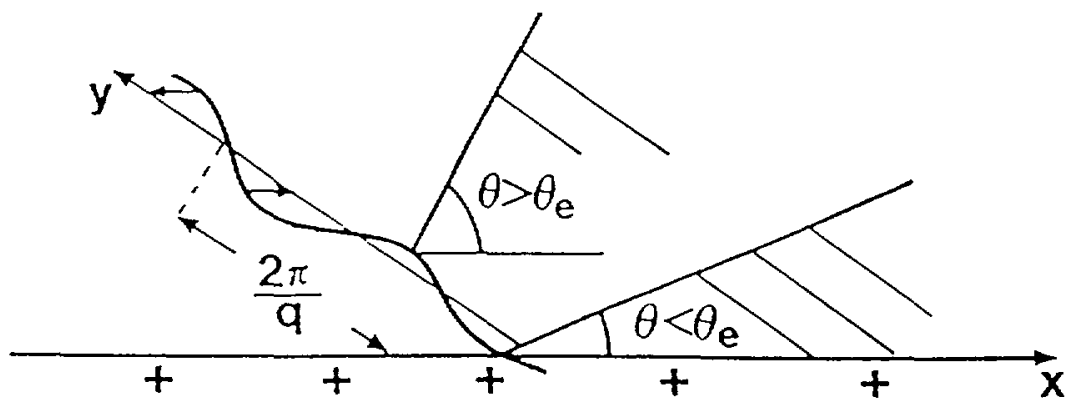

Fig. 2. - "Triplon" modes of a contact line.

Equation (8) holds when gravitational energies are negligible $(q>\kappa)$. In this regime, the line elasticity cannot be described in terms of a line tension. A tension $\zeta$ would generate an energy $\sim \zeta q^{2}\left|u_{q}\right|^{2}$ The distortions of the liquid-vapor interface near the contact line extend (in the direction normal to the line) up to distances of order $q^{-1}$ This is the source of the anomalous factor $|q|$ in equation (8). In the opposit limit $(q<\kappa)$, the deformations are screened at a distance $\kappa^{-1}$, and the energy has a standard form:

$$
f_{\mathrm{el}} \mathrm{q}=\frac{1}{4} \gamma \theta_{\mathrm{E}}^{2} \kappa^{-1}|q|^{2}\left|u_{q}\right|^{2}
$$

The general expression interpolating between these two limits has been derived more recently $[16]$ :

$$
f_{\mathrm{e} 1 \mathrm{q}}=\frac{1}{2} \gamma \theta_{\mathrm{E}}^{2}\left(\sqrt{\kappa^{2}+q^{2}}-\kappa\right)\left|u_{q}\right|^{2}
$$

We derive the dynamical features of the mode from the balance between the elastic restoring force deduced from equation (10) and the inertial force:

$$
\gamma \theta_{\mathrm{E}}^{2}\left(\sqrt{\kappa^{2}+q^{2}}-r\right)\left|u_{q}\right|=M \omega^{2} u q
$$

where $M$ is the mass per unit length of the moving liquid and $\omega$ the frequency of wave. 
3.1 CAPILlaRY REgIME $(q>\kappa)$. - In this limit, the perturbation extends over a region of size $q^{-1}$ normal to the direction of the line, and the scaling form of $M$ is:

$$
M=\rho \frac{\theta_{\mathrm{E}}}{q^{2}}
$$

From equation (11), we find the frequency $\omega(q)$ of the propagative mode omitting an exact numerical coefficient:

$$
\omega(q)=q^{3 / 2} \sqrt{\frac{\gamma}{\rho} \theta_{\mathrm{E}}} .
$$

Joanny has calculated the frequency of the oscillation of superfluid droplets around their equilibrium shape [17] and finds $\omega=\sqrt{\frac{\gamma \theta_{\mathrm{E}}}{\rho R_{\mathrm{e}}^{3}}}$ in agreement with equation (13).

3.2 GRAVITY REGIME $q<\hbar^{-1} \quad$ - For long wavelengths, the deformation extends on the capillary length $\kappa^{-1}$ and the mass $M$ is now:

$$
M \sim \rho \theta_{\mathrm{E}} \kappa^{-2}
$$

Equation (10) leads to standard propagative modes of a vibrating rope $\omega=c q$, where the velocity of the wave, $c$, is:

$$
c=\sqrt{\frac{\gamma \theta_{\mathrm{E} \kappa}}{2 \rho}}=\sqrt{g \frac{\kappa^{-1}}{2} \theta_{\mathrm{E}}},
$$

with $\kappa^{-1} \sim 0.5 \mathrm{~mm}, \theta_{\mathrm{E}} \sim 10^{-1}$, one finds $c \sim \mathrm{m} . \mathrm{s}^{-1}$

\section{Concluding remarks.}

We have studied some dynamical features of superfluid ${ }^{4} \mathrm{He}$ deposited on non-wettable substrates at very low temperatures, where the role of vapor is negligible.

i) Thin films are unstable and dewet by nucleation and growth of dry patches, which open at constant velocity $U=U^{*} \theta_{\mathrm{E}}$, where $U^{*}=\sqrt{g \frac{\hbar^{-2}}{e}}$. Contrary to the case of viscous liquids where the dewetting velocity was independent of the initial film thickness $e$, one expects $U \sim e^{-1 / 2}$ for inertial liquids. By video recording the opening of micro or macro dry patches, one can monitor the film thickness varying over a broad range $(100 \AA-100 \mu \mathrm{m})$. The metastable films observed recently by liutledge and Taborek [5] do not dewet spontaneously. However, if a hole in the film is formed (by a laser beam), the film will dewet probably by the mechanisms described here.

ii) The "triplons" modes of the contact line are propagative. As for capillary waves of usual liquids in the inertial regime, we find $\omega \sim q^{3 / 2}$ for large wave vectors $q$. On the other hand, for undulations of small wave vectors, the deformations are screened and $\omega=c q$, where $c \sim \sqrt{g \kappa^{-1}}$. 


\section{Acknowledgements.}

We thank P.G. de Gemnes and J.F. Joanny for stimulating discussions on the wetting of ${ }^{4} \mathrm{He}$, C. Redon on dewetting and the Referee for his useful comments.

\section{References}

[1] Cheng E., Cole M.W., SaAm W.F. and Treiner J., Phys. Rev. Lett. 67 (1991) 1007.

[2] NACher P.J. and DuPont-roc J., Phys. Rev. Lett. 67 (1991) 2966.

[3] Mukherjee S.K., Druist D.P. and Chan M.H.W., J. Low Temp. Phys. 87 (1992) 113.

[4] Ketola K.S., Wang S. and Hallock R.B., Phys. Rev. Lett. 68 (1992) 201.

[5] Rutledge J.E. and Taborek P. Phys. Rev. Lett. 69 (1992) 937.

[6] Redon C., Brochard-Wyart F. and Rondelez F., Phys. Rev. Lett. 66 (1991) 715.

[7] Brochart-wyart F., di Meglio J.M. and Queré D., C. R. Acad. Sci. (Paris) II 304 (1987) 553.

[8] Brochard-wyart F. and Daillant J., Can. J. Phys. 68 (1990) 1084.

[9] Brochard-wyart F., Redon C. and Sykes C., C. R. Acad. Sci. (Paris) 314 (1992) 131.

[10] Reiter G., Phys. Rev. Lett. 68 (1992) 75.

[11] ONDARCUHU T. and VeYsSiÉ M., Nature 352 (1991).

[12] BRochaRD-WYART F. and DE GENNES P.G., Langmuir 7 (1991) 3216.

[13] Culick F.E.C., J. Appl. Phys. 31 (1960) 1128.

[14] Mysels K.J. and ViJayendRan B.R., J. Phys. Chem. 81 (1977) 731.

[15] Joanny J.F. and DE Gennes P.G., J. Chem. Phys. 81 (1984) 552.

[16] Sekimoto K., Oguma R. and Kawasaki K., Ann. Phys. 176 (1987) 359.

[17] JoANnY J.F., J. Phys. France 46 (1985) 807.

[18] Levich V., Physical Hydrodynamics (Prentice Hall N.J., 1962). 